\title{
LITERATUR
}

\author{
Aart van de Laar
}

\section{The World Bank and the Poor}

Institute of Social Studies, Series on the Development of Societies, Vol. VI, Martinus Nijhoff Publishing, Bosten, The Hague-London 1980, 269 S., US $\$ 15.00$

Neben den beiden ebenfalls 1980 erschienenen Weltbank-Studien von Bettina Hürni (Schweiz) und Rainer Tetzlaff (Bundesrepublik Deutschland) ist diese Arbeit des Holländers van de Laar die dritte aktuelle Studie, die sich mit der Entwicklungspolitik der Weltbank beschäftigt. Den Fachleuten war der ehemalige Mitarbeiter der Weltbank schon aus einigen kritischen Aufsätzen zur "armutsorientierten" Entwicklungsstrategie der Weltbank in der McNamara-Ära bekannt, jetzt hat von de Laar seine massive Weltbankkritik noch einmal sehr kenntnisreich und detailliert vorgetragen, freilich ohne das Kind mit dem Bade auszuschütten (wie dies vor zehn Jahren etwa Teresa Hayter mit ihrem wichtigen, aber doch partiell überzogenen Buch "Aid as Imperialism" getan hatte).

Die Studie umfaßt die Geschichte von Weltbank und ihrer Tochterorganisation für die ärmsten Entwicklungsländer, die International Development Association (IDA), ferner eine Charakterisierung der Arbeitsweise, Rekrutierung und Erfolge des Weltbankpersonals, eine Analyse der Kreditvergabe der Bank nach dem Kriterium der Armut der Empfängergruppen sowie schließlich ein gesondertes Kapitel über die gegenwärtige und zukünftige Organisation der Weltbank nach dem Maßstab Zentralisierung/Dezentralisierung von Macht, Kontrolle und entwicklungspolitischen Entscheidungen.

Während van de Laar viele Informationen wiedergibt, die auch schon in früheren Büchern über die Weltbank vorkommen (vor allem in dem Standardwerk von Mason/Asher oder in den Jahresberichten und Policy Papers der Weltbank enthalten sind), ist das 6. Kapitel über die Kleinbauernprojekte der Weltbank von besonderer Aktualität und Originalität: Hier beschreibt ein guter Kenner der komplexen Materie ländlicher Entwicklungshilfe die internen und externen Schwierigkeiten beim ehrgeizigen Ziel, mittels integrierter Förderungsprogramme Kleinbauern in Armutsländern erreichen zu wollen. Uberall stießen auch die an Landreformen interessierten Weltbank-Mitarbeiter auf die Widerstände der lokalen Machteliten, die in der Regel die von außen kommenden Hilfsressourcen nach ihrem Eigeninteresse manipulieren können, so daß die eigentlichen Zielgruppen - die hart arbeitenden Kleinst- und Kleinbauern - doch wieder leer ausgingen. Teilweise wird sogar als Folge gut gemeinter, aber nicht realisierbarer Modernisierungsprogramme das Los der Kleinbauern noch schlechter als zuvor. Ferner weist van de Laar darauf hin, wie durch die modernen, von der Weltbank mitfinanzierten Saatgut-DüngerTechnologien die Abhängigkeit der Landbewirtschafter in der Dritten Welt von auswärtigen Lieferungen (was Preis, Menge und Pünktlichkeit angeht) gefährlich anwächst. 
Insgesamt handelt es sich hier um ein kompetent geschriebenes, bei aller Parteilichkeit informatives Buch, das weniger entwicklungstheoretisch, als eher entwicklungspraktisch interessant ist. Es thematisiert die notwendige Kritik an den in drei Jahrzehnten entstandenen paternalistischen Verhältnissen zwischen der Weltbank und ihren Schuldnerländern - unter anderem die politische Unkontrollierbarkeit der Weltbankaktivitäten sowie die weltbankspezifischen Widersprüche zwischen großartiger politischer Rhetorik als Anwalt der Ärmsten und der eher mittelmäßigen Realität bei der Verwirklichung der weitgesteckten Ziele. Deutlich wird hier vor allem, wie schwierig generell die Erreichung von sozial progressiven Entwicklungszielen in den ländlichen Räumen von Entwicklungsländern ist. Auch wenn der Autor selbst keine alternativen Entwicklungsstrategien anbieten kann (zum Glück, denn die gibt es pauschal auch gar nicht) und sich mit einigen interessanten Erwägungen über die regionale Dezentralisierung der Riesenbehörde in Washington begnügt, so ist dieses in kritischer Solidarität mit der internationelen Entwicklungshilfe geschriebene Buch eine verdienstvolle Arbeit über die Weltbank und die Probleme der internationalen Entwicklungspolitik, - kurzum eine ebenso kritische wie kompetente Studie, die allen Dritte-Welt-Interessierten empfohlen werden kann.

Rainer Tetzlaff

\section{A. Peter Mutharika (Herausg.)}

The International Law of Development Basic Documents, Vol. 1

Oceana Publications, Inc., Dobbs Ferry, New York, 1978, 646 S.

Bei diesem Dokumentenband handelt es sich um die ersten drei Kapitel (1. Band) einer auf 17. Kapitel (insgesamt 4 Bände) angelegten Sammlung von Verträgen und Gesetzestexten zum Thema internationale Entwicklung. Dieser erste Band, der von Peter Mutharika, Professor für Recht an der Universität von Washington, besorgt wurde, beschränkt sich auf die Wiedergabe von Verträgen und Resolutionen zu folgenden drei thematischen Bereichen. Zuerst werden die Statuten der Weltbank und ihrer Tochterorganisationen (IDA und IFC), der Regionalen Entwicklungsbanken für Lateinamerika, Asien und Afrika reproduziert sowie die Gründungsstatuten von subregionalen Banken wie z. B. der 1969 gegründeten Karibischen Entwicklungsbank. Das 2. Kapitel behandelt die Verfahrensweisen und Ausführungsbestimmungen all dieser Entwicklungsbanken. Dabei ist das Kreditabkommen zwischen der Weltbank und der Regierung von Indien aus dem Jahr 1976 hervorzuheben (S. 317-350), das einen detaillierten Einblick in die allgemeine Kreditvergabepraxis der Weltbank (Konditionen, Definitionen von Nutznießern, politische und finanzielle Auflagen etc.) gewährt.

Das knapp hundert Seiten umfassende dritte Kapitel stellt den Beginn der Serie von Resolutionen der Vereinten Nationen über Entwicklungsprobleme dar. Die wichtigsten hier im 1. Band wiedergegebenen Dokumente sind die Resolutionen der UN-Generalver- 